\title{
Anatomy and Diseases of Human Biliary System: An Analysis by Mathematical Model
}

\author{
Dharna Satsangi \\ Department of Mathematics, Dayalbagh Educational Institute, Agra, India \\ Email: dharna.satsangi@gmail.com \\ Arun K. Sinha \\ Department of Mathematics, Dayalbagh Educational Institute, Agra, India \\ Email: arunsinha47@gmail.com
}

\begin{abstract}
The objective of this paper is to develop an understanding of the diseases related with gallbladder, liver, and biliary tract. The study focuses on human biliary system that is how bile flows in the human body. This can be done by developing an understanding of gallbladder and bile flowing in the body and related organs very briefly. Gallstone is an important disease of gallbladder and is closely related to pressure drop. A small model for the human biliary system is also analyzed in this study. The cylindrical model of gallbladder and ducts in contraction and extension phase is used for the study. The amount of substances present in the organ varies in these cases. With the help of this study it is concluded that the flux decreases on increasing the radius and length of the cylinder. It is observed that the behavior of flow of bile in gallbladder is similar to the flow of bile in the ducts.
\end{abstract}

Index Terms - Biological system modeling, Differential equations, Human biliary system, Disease

\section{INTRODUCTION AND HUMAN BILIARY SYSTEM}

Since ages man is trying to know about the basic functionality of human body. There are several functions of human body such as respiration, digestion, excretion etc. Along with these functions there is a function of flowing bile presented by Burget [1] and Rodkiewicz [2] respectively in 1925 and 1978 . After their studies several researchers started working in this area. Although all the functions of human body seem to be different but somewhere they are connected with biliary system therefore first we go on describing biliary system.

The human biliary system consists of the organs and ducts (bile ducts, gallbladder etc.), which are involved in the production and transportation of bile. These organs are liver, biliary tract, and gallbladder.

\section{The liver}

\section{Anatomy:}

The liver lies to the right of the stomach and overlies the gallbladder. The human liver in adults weighs between
1.4-1.6 kilograms (3.1-3.6 pounds). It is a soft, pinkishbrown, triangular organ. It is both the largest internal organ and the largest gland in the human body. The liver is a vital organ present in vertebrates and some other animals. A human can last up to 24 hours without liver function. It plays a major role in metabolism and has a number of functions in the body. It produces bile, an alkaline compound which aids in digestion, via the emulsification of lipids. It also performs and regulates a wide variety of high-volume biochemical reactions requiring very specialized tissues.

\section{Functions:}

The Liver produces and excretes bile required for emulsifying fats. Some of the drains directly into the duodenum, and some are stored in the gallbladder. It also plays several roles in carbohydrate metabolism and lipid metabolism. The conversion of ammonia to urea is performed by the liver. It produces the major osmolar component of blood serum, albumin.

\section{The Biliary Tract}

It begins with many small branches which end in the common bile duct, referred as trunk of the biliary tree. It is divided in three parts:

- Cystic duct

- Common hepatic duct

- Common bile duct

The biliary tract (biliary tree) is the common anatomy term for the path by which bile is secreted by the liver on its way to the abdomen or small intestine. It is referred to as a tree because it begins with many small branches end in the common bile duct, sometimes referred to as the trunk of the biliary tree. Bile flows in opposite direction to that of the blood present in the other two channels.

\section{Cystic duct}

The cystic duct is the short duct that joins the gallbladder to common bile duct. It usually lies next to the cystic artery. It ranges from 2 to $5 \mathrm{~mm}$ and its length from 10 to $60 \mathrm{~mm}$. The number of folds varies between 2 
to 14 . The human cystic duct functions for the transport of bile during both filling and emptying the gallbladder.

\section{Common hepatic duct}

The common hepatic duct is formed by the convergence of the right hepatic duct and the left hepatic duct. The common hepatic duct then joins the cystic duct coming from the gallbladder to form the common bile duct. It measures usually $6-8 \mathrm{~cm}$ in length and approximately $6 \mathrm{~mm}$ wide in adults. The hepatic duct transports more volume in the people who have had their gallbladder removed.

\section{Common bile duct}

The duct that carries bile from the gallbladder and liver into the duodenum is called common bile duct. The common bile duct is formed by the function of the cystic duct that comes from the gallbladder and the common hepatic duct that comes from the liver.

\section{The Gallbladder}

\section{Anatomy:}

The Gallbladder is connected to the liver and the duodenum by the biliary tract. The cystic duct connects the gallbladder to the common hepatic duct to form the common bile duct. The common bile duct the joins the pancreatic duct and enters through the hepatic pancreatic ampulla at the major duodenal papilla.

The gallbladder is a pear or oval shaped digestive organ located under the right side of the liver, and connected to the small tubes called bile ducts and to the small intestine through which bile flows. It is about 3-4 inches long in humans and appears dark green because of bile. It stores and concentrates bile and helps in digestion of fats. Every day the liver produces almost a liter of bile and the gallbladder helps in storing and concentrating. The hormone causes the Gallbladder to contract, joining bile into the common bile duct. A valve which opens only when food is present in the intestine, allows bile to flow from the common bile duct into duodenum. Sometimes the substances contained in bile crystallize in the Gallbladder forming Gall stones, a disease of gallbladder.

\section{Function:}

Bile is continually being made and secreted by the liver into bile ducts in varying amounts. Some of it goes directly into the small intestine and into the gallbladder. The gallbladder stores the bile to help to breakdown the fats of fatty meals. It also acts as a reservoir that uptakes excess bile when there is pressure in the bile ducts.

\section{Necessity of the gallbladder}

The Gallbladder seems to be a vestigial organ. Indeed, gallbladders have been removed from people for over one hundred years without any known side effects. However if one eats a particularly rich and or fatty meal, some degree of diarrhea may result.

\section{Bile}

Bile is important in digestion. It is produced by the liver and is poured into the intestine through the bile duct but the amount varies with the diet. The evacuation of the gallbladder is controlled by a hormone called cholecystokinin (CCK). It is a viscid liquid, alkaline in reaction and bitter in taste, having a color from yellowish brown to green. The bile is a secretary and an excretory product. The bile salts which are important in the digestion and absorption of fats should be considered a secretion and the bile pigment and cholesterol, which apparently have no function in the intestine and are largely eliminated from the body. Bile is the complex fluid composed of bile salts, cholesterol and other molecules. The bile salts are the break down product of hemoglobin. Bile salts and bile itself are formed in the liver and excreted into bile ducts which converge in the liver to form the main bile ducts. The common bile duct enters the duodenum, the earliest part of the small intestine where digestion and absorption of food begins. Normal man makes $1000-1500$ cc of bile per day. Some amount of bile entering our intestinal tract goes into the gallbladder as it comes down the duct. About half of the bile secreted between meals flows directly through the common bile duct into the small intestine. The rest of the bile is diverted through the cystic duct into the gallbladder to be stored. In the gallbladder, up to $90 \%$ of the water in bile is absorbed into the blood stream, making the remaining bile very concentrated.

\section{Functions:}

The bile has two major functions in the body. It breaks down the fats that we eat. Without adequate bile we do not metabolize our fats well which can result in a deficiency of the fat-soluble vitamin A, D, E and K. There may be problems digesting the essential fatty acids without bile. The symptoms include dry skin, peeling on the soles of our feet, indigestion of fats, nauseous or experience gas and bloating. It is also a very powerful antioxidant which helps to remove toxins from the liver.

After bile enters and passes down the small intestine, about $90 \%$ of bile salts are reabsorbed into the bloodstream through the wall of the lower small intestine. The liver extracts these bile salts from the blood and resecretes them back into the bile. Bile salts through this cycle about 10 to 12 times a day. Each time, small amounts of bile salts absorb and reach large intestine, where they are broken down by bacteria. Some bile salts are reabsorbed in the large intestine. The rest are excreted is the stool.

\section{Necessity:}

We need bile to absorb fats. Our intentional living can absorb water but not fats. Since fat is not dissolvable in water we cannot absorb fats unless something makes the 
water and fats attach. It can bind to both water and fat. Therefore when we absorb water, the fat absorb with it if bile is present to link the water to the fat. If we do not have any bile we will not be able to absorb fats.

\section{Functions of bile}

It aids in digestion and eliminates certain waste products like excess cholesterol.

$90 \%$ of bile salts are reabsorbed into the bloodstream through the wall of the lower small intestine after entering the small intestine.

\section{Composition of bile:}

The composition of bile secreted by the liver and that collected from gallbladder differs. Its composition varies from time to time since certain of the biliary constituents represent material in the process of excretion from the body.

\section{Bile pigments:}

The main pigment in the body is hemoglobin of the blood. This is a compound protein, composed of the protein globins and the red-colouring matter hems, Hemoglobin is contained in the R.B.C. When the average life of R.B.C. is completed, hemoglobin is liberated and is converted into the bile pigments, biliverdin and bilirubin. These pigments are derived from the heme portion of hemoglobin. The bile pigments contain no iron Thus before heme is discarded by the body, its iron is removed and used again in the synthesis of hemoglobin. Biliverdin is the original bile pigment that is green. In some animals, including man, much of the biliverdin is reduced to bilirubin, which is yellow, and imparts the yellow colour to the bile. The occasional production of green faces in infants with diarrhea is due to biliverdin and indicates that oxidation, instead or normal reduction, has taken place in the intestine. If there is an obstruction to the flow of bile or liver function is impaired, or if R.B.C are being destroyed more rapidly than normal, bile pigments accumulate in the blood and the skin will become yellow. This condition is known as jaundice.

\section{Bile Salts:}

The main bile salts are the sodium salts of glycocholic and taurocholic acid. These acids are complicated structure, composed of glycol and taurine in combination with cholic acids. Glycol is another name for the simplest amino acid; glycin. Taurine is the amino acid cysteine with the carboxyl group removed and with the sulphur oxidized from a valency of 2 to 6 .

The bile salts are the most important constituents of the bile from the standpoint of digestion in the presence on the salts; the activity of the pancreatic lipase is greatly increased. This increase in activity appears to be due to the emulsification of fats in the presence of the bile salts. Bile salts aid in emulsification of fats. Another important function of the bile salts is to aid in the absorption of fatty acids. Fatty acids in combination with bile salts are soluble and can be absorbed through the intestinal wall in this form. The bile salts, thus reabsorbed, are carried to the liver, where they are again secreted in the bile. Thus, there is a circulation of the bile salts in the body.

By aiding fat digestion bile salts also aid indirectly digestion of other food constituents. If fat is present in food in large quantities, it may cost other food particles, thus preventing the action of other enzymes.

\section{Cholesterol:}

The third important constituent of the bile is the cholesterol. Cholesterol in the bile is considered a waste product, which is removed from the body through the liver. A reduced product of cholesterol called coprosterol, is derived from cholesterol in the intestine.

Precipitation of cholesterol in the liver in large quantities results in the formation of stones in the gallbladder. Several theories have been put forth concerning the origins of gall stones. One is that they are a result of infection in the gallbladder. When infection occurs, nuclei are produced upon which cholesterol deposits forming stones. Another theory is that they are produced due to faulty metabolism. Cholesterol is held in solution in the bile by means of bile salts. If too much cholesterol is excreted in the bile or if the concentration of the bile salts is low, cholesterol may precipitate. A third theory is that they are a result of stagnation of the bile in the gallbladder. When this occurs bile salts may be reabsorbed into the bold stream, leaving the cholesterol without sufficient bile salts to hold it in solution.

Major components of bile by approximate weight include several components. They are shown in the Table1:

Table1: Components of bile by weight in percentage

\begin{tabular}{|c|c|}
\hline Components & $\begin{array}{c}\text { Percentage } \\
(\%)\end{array}$ \\
\hline Water & 82.0 \\
\hline Bile acids & 12.0 \\
\hline Lecithin and phospholipids & 4.0 \\
\hline Unsterilized cholesterol & 0.7 \\
\hline $\begin{array}{c}\text { Conjugated bilirubin, protein, electrolytes, } \\
\text { mucus }\end{array}$ & 1.3 \\
\hline
\end{tabular}

\section{DISEASES OF GALLBLADDER}

\section{Related diseases of gallbladder}
1. Gallstones
2. Acute and Chronic Cholecystitis
3. Jaundice
4. Cancer of the Gallbladder

\section{Gall stones}

Gallstones are crystalline structures formed by concretion or accretion of normal or abnormal bile 
constituents. These are mostly found in most western countries. In the Unites States series have shown gallstone over the age of 40 . It is estimated that 16 to 20 million persons in the Unites States have gallstones and that approximately one million new cases of cholelithiasis develop each year [12].

\section{Causes:}

Scientists believe cholesterol stones from when bile contains too much cholesterol, too much bilirubin, or not enough bile salts, or when the gallbladder does not empty completely or often enough.

The mere presence of gallstones may cause more gallstones to develop. Other factors that contribute to the formation of gallstones, particularly cholesterol stones include:

- Age: People more than 60 years are more likely to develop gallstones than younger people. As people age, the body tends to secrete more cholesterol into bile.

- Diet: Diet high in fats and cholesterol and low in fiber increase the risk of gallstones due to increased cholesterol in the bile and reduced gallbladder emptying.

- Diabetes: People with diabetes generally have high levels of fatty acids called triglycerides. These fatty acids may increase the risk of gallstones.

- Family: Gallstones often run in families, pointing to a possible genetic link.

- Weight: A study showed that overweight persons have the risk for developing gallstones. The most likely reason is that the amount of bile salts in bile is reduced, resulting in more cholesterol. Increased cholesterol reduces gallbladder emptying. Obesity is a major risk factor for gallstones, especially in women. It also occurs after rapid weight loss. As the body metabolizes fat during prolonged fasting and rapid weight loss- such as "crash diets"- the liver secretes extra cholesterol into bile, which can cause gallstones. In addition, the gallbladder does not empty property.

- Sex: Women are twice as likely as men to develop gallstones. Excess estrogen from pregnancy, hormone replacement therapy, and birth control pills appears to increase cholesterol levels in bile and decrease gallbladder movement, which can lead to gallstones.

\section{Symptoms:}

Its symptoms include steady pain in the right upper abdomen that increases rapidly and lasts from 30 minutes to several hours, pain in the back between the shoulder blades, pain under the right shoulder, prolonged pain - more than 5 hours, nausea and vomiting, fever - even low grade - or chills, yellowish color of the skin or whites of the eyes, clay colored stools etc.

\section{Treatment:}

Surgery for removal of the gallbladder.

\section{Acute and Chronic Cholecystitis}

a) Acute Cholecystitis: Acute inflammation of the gallbladder wall usually follows obstruction of the cystic duct by a stone.

\section{Symptoms:}

Its symptoms include persistent pain and a temperature lasting more than 12 hours, pain and tenderness under the ribs on the right side, pain that is made worse by movement or coughing etc.

b) Chronic cholecystitis: Chronic inflammation occurs due to repeated bouts of sub acute cholecystitis. The presence of bacteria in the bile occurs in more than one quarter of patients with chronic cholecystitis.

\section{Symptoms:}

Its symptoms include sporadic pains in the middle of the upper abdomen, or just below the ribs on the right side, pain which becomes worse over an hour and then stays the same, pain that may spread to the right shoulder or between the shoulder blades, pain that can be accompanied by nausea and vomiting and sometime excessive wind etc.

\section{Treatment:}

A course of antibiotics is advised for it. If this is not effective, surgery may be required to remove the gallbladder.

\section{Jaundice (yellow discoloration of the skin and whites of eyes)}

This is caused when there is an obstruction to the flow of bile from the liver.

\section{Symptoms:}

Its symptoms include increasingly yellow eyes and skin, skin can become itchy, pale bowel motions and dark urine etc.

\section{Cancer of the Gallbladder}

Most cancers of the gallbladder develop in conjunction with stones. In patients with gallstones, the risk for developing gallbladder cancer, while increased, is still quite low. In one study, gallbladder cancer developed in only 5 of 2583 patients with gallstones followed for a medium of 13 years. In the United States, adenocarcinomas comprise the vast majority of the estimated 6500 new cases of gallbladder cancer 
diagnosed each year. The female/male ratio is $4: 1$ and the mean age at diagnosis is approximately 70 years.

Cancer of the gallbladder is rate. It is more common in women and Native Americans.

\section{Symptoms:}

Its symptoms include jaundice (yellow of the skin and whites of the eyes), pain above the stomach, fever, nausea and vomiting, bloating etc.

\section{Treatment:}

- Chemotherapy

- Radiation Therapy

- Surgery

\section{DISEASES OF BILIARY TRACT}

\section{Choledocholithiasis}

Choledocholithiasis is the common causes of extrahepatic biliary obstruction.

Stones in the bile duct can cause biliary obstruction and cholestasis. This can lead to infection in the bile duct, which requires urgent medical therapy. The long-standing presence of stones in the bile duct can lead to secondary biliary cirrhosis. Choledocholithiasis can also lead to gallstone pancreatitis.

\section{Signs and Symptoms:}

Upper abdominal pain, episodic jaundice, pancreatitis, epigastric pain, nausea, and vomiting.

\section{Treatment:}

Stone extraction, endoscopic sphincterotomy.

\section{Cholangiocarcinoma}

Cholangiocarcinoma is an adenocarcinoma of the intrahepatic or extrahepatic bile duct.

\section{Signs and Symptoms}

Jaundice, weight loss, anorexia, and fatigue.

\section{Treatment:}

Radiation and chemotherapy followed by liver transplantation in patients with early-stage disease.

\section{LITERATURE REVIEW}

Burget [1] explained the physiology of the gallbladder, and resistance of the sphincter of Oddi have attracted attention largely from a surgical point of view. Taylor et al. [5] described that the factors influencing the migration of gall stones are ill understood. Altogether 331 patients undergoing cholecystectomy were studied prospectively. The diameters of the cystic and common bile ducts and of stones in the gall bladder and bile ducts were measured.
Pressure increasing was applied to the freshly excised gall bladder in an attempt to evacuate stones through the cystic duct. Along with this Jungst et al. [6] explained that super saturation and rapid nucleation of cholesterol in bile are of key importance in the pathogenesis of cholesterol gallstones. While the effects of bile acids and phospholipids on cholesterol saturation of bile have been extensively studied, their influence on the cholesterol nucleation time has not been compared. The increase in the phosphatidylcholine or bile acid concentration decreased the mean cholesterol saturation index to a similar extent. Rodkiewicz et al. [3] concluded that the anatomical and physiological aspects of the flow of bile through the extrahepatic biliary system are quite well known and understood; little is known about the physical behavior of bile from a Fluid Mechanics point of view. Bile proves to be an interesting fluid flow case. Experiments are made showing that bile, flowing in a rigid tube, behaves like a Newtonian fluid. From this, one might predict that bile will also behave like a Newtonian fluid in the human biliary tree. The immediate and permanent consequence available from Fluid Mechanics and Heat Transfer principles, applicable to the laminar flow of Newtonian fluids, may be used in seeking the qualitative and quantitative understanding of the flow phenomena in the human biliary tree. Mazer et al. [4] proposed a mathematical model of biliary lipid secretion. In this model coupling of secretion rates of bile salts, lecithin, and cholesterol into bile were taken into account. The four independent parameters related to the postulated mechanisms of biliary lipid "secretion parameters" also determine how the biliary lipid composition of hepatic and "fasting' gallbladder bile varies with the bile salt secretion rate. A quantitative analysis of biochemical and physiological data on biliary lipid secretion in rat, dog, and man confirms the general predictions of the model. Deductions of the secretion parameters are made for each species and are compared with other relevant data on biliary lipid metabolism. Rodkiewicz et al. [2] considered flow of bile in the biliary tree. On the grounds of experiments performed on dogs, it is found that the rate of flow of bile in a duct of the extrahepatic biliary tree is related to the associated pressure drop by the power law

$$
Q=a \frac{\pi D^{2}}{128 \mu}\left(\frac{\Delta p}{L}\right)^{b}
$$

where $a$ and $b$ are constants dependent on the segment considered; $D$ is the duct diameter; $L$ is the duct length; and $\mu$ is the bile viscosity. Jungst et al. [7] aimed that an increased viscosity of gallbladder bile has been considered an important factor in the pathogenesis of gallstone disease. Besides lipids and proteins, mucin has been suggested to affect the viscosity of bile. To further clarify these issues they compared mucin, protein and the lipid components of hepatic and gallbladder bile and its viscosity in patients with gallstones. Hence, concluded that the viscosity of gallbladder bile is markedly higher than that of hepatic bile in patients with gallstones. Dang et al. [8] explained that biliary duct obstruction is an important clinical condition that stems from cholelithiasis, the neoplasm in the wall commonly known as gallbladder 
stones. The objective of this study is to understand the structural and mechanical remodeling of the common bile duct (CBD) post obstruction. Bird et al. [9] started their study with the cystic duct because the anatomy of the duct is one of the factors responsible for formation of gallstones. It allows low viscosity hepatic bile to enter the gallbladder under low pressure and expulsion of more viscous gallbladder bile. Under this paper there is discussion of variation in geometry of the cystic duct, obtained from acrylic resin casts of the neck and first part of the cystic duct in gallbladders removed for gallstone disease and obtained from patients undergoing partial hepatectomy for metastatic disease. In this paper the term "spiral valve" is explained and then spiraling was dominant feature of cystic duct. Li et al. [10] were interested in the pressure drop in biliary system. They proposed that excessive pressure drop during bile emptying and refilling may result in incomplete bile emptying. This leads to stasis and subsequent formation of gallbladder stones. Li et al. [11] extended their study of human biliary system to include two important factors: the non-Newtonian properties of bile, and elastic deformation of the cystic duct.

\section{FORMULATION AND SOLUTION OF THE MODEL}

\section{Mathematical Model}

W.G. Li et.al. used the model for pressure drop while emptying. And this is directly related with the formation of gallstones present in gallbladder.

In this model the shape of ducts is considered to be cylindrical and T-junction is where common bile duct and common hepatic duct joins. In order to find compatibility of gallbladder with these ducts we need to analyze cylindrical model of gallbladder which deals with the flow of bile flowing in the gallbladder and finally pressure difference. For analyzing this study with their study it is important to use same nomenclature. Hence taking a particular case that pressure drop is a special case of pressure difference, the model is analyzed. First we solve cylindrical model of gallbladder.

Initially we considered an idealized cylindrical model of gallbladder. The pressure with the common duct exceeds any resistance to flow through the cystic duct and therefore bile enters the gallbladder. During this period, the secretary rate of the liver is minimal for the delivery of bile into the duodenum.

Assuming that the bile flow is slow, symmetric and quasi-steady, the basic equations governing as motion due to periodic radial dilation of the gallbladder are written in the cylindrical polar coordinates $(r, \theta, z)$ as follows:

$$
\mu\left\{\frac{\partial^{2} u_{z}}{\partial r^{2}}+\frac{1}{r} \frac{\partial u_{z}}{\partial r}\right\}=\frac{\partial P}{\partial z}
$$

$$
\frac{\partial}{\partial r}\left(r u_{r}\right)+\frac{\partial}{\partial z}\left(r u_{z}\right)=0
$$

where, $u_{r}$ and $u_{z}$ are radial and axial components of the velocity, $P$ the pressure and $\mu$ is the viscosity assumed to be constant. It may be noted that the pressure gradient $\frac{\partial P}{\partial z}$ essentially does not vary across the radius.

The following boundary conditions may be prescribed for (1) and (2):

$$
\begin{array}{ll}
u_{z}=0 & r=R(z, t) \\
u_{r}=\frac{\partial R}{\partial t} & r=R(z, t) \\
\frac{\partial u_{z}}{\partial r}=0 & r=0 \\
u_{r}=0 & r=0
\end{array}
$$

where, $R(z, t)$ is the instantaneous radius of the gallbladder at time t. The boundary conditions (3) and (4) imply that the velocity components are continuous at the wall of the gallbladder while (5) and (6) suggests that the axial velocity is maximum at the axis and the radial component is zero. Integrating (1) and using the boundary conditions (3) and (5) we get

$u_{z}=\frac{1}{4 \mu} \frac{\partial P}{\partial z}\left(r^{2}-R^{2}(z, t)\right)$

Determining the volume flow rate by

$Q(z, t)=\int_{0}^{R(z, t)} 2 \pi r u_{z} d r$

And using (7) we get

$Q(z, t)=-\frac{1}{8 \mu} \pi \frac{\partial P}{\partial z}\left(R^{4}(z, t)\right)$

Integrating (2) and using boundary condition (4), (6) and (8) we get the equation determining the flow rate

$$
\frac{\partial Q}{\partial z}=-2 \pi R(z, t) \frac{\partial R(z, t)}{\partial t}
$$

From (9) and (10) we obtain the following equations for determining the pressure

$$
\frac{\partial}{\partial z}\left(R^{4}(z, t) \frac{\partial P}{\partial z}\right)=16 \mu R(z, t) \frac{\partial R(z, t)}{\partial t}
$$

Equation (10) and (11) determine the flow rate and the pressure and are related through radius $\mathrm{R}$.

Again using boundary conditions where $0 \leq z \leq L$ 


$$
\begin{array}{llll}
Q(z, t)=0 & \text { at } & & z=0 \\
Q(z, t)=Q_{L} & \text { at } & z=L \\
P(z, t)=P_{0} & \text { at } & z=0 \\
P(z, t)=P_{L} & \text { at } & z=L
\end{array}
$$

Integrating (10) and equating with (9) we get,

$Q(z, t)=\int_{0}^{z}-2 \pi R(z, t) \frac{\partial R}{\partial t} d z+c_{1}$

Since at $z=0, Q(z, t)=0$

Therefore, $c_{1}=0$

Hence, $Q(z, t)=-2 \pi R(z, t) \frac{\partial R(z, t)}{\partial t} z$

Now equating with (9)

$-\frac{\pi}{8 \mu} R^{4} \frac{\partial P}{\partial z}=-\int_{0}^{z} 2 \pi R \frac{\partial R}{\partial t} d z$

Hence,

$$
\begin{aligned}
& -\frac{\partial P}{\partial z}=-\frac{8 \mu}{\pi R^{4}} \int_{0}^{z} 2 \pi R \frac{\partial R}{\partial t} d z \\
& -P=-\int_{0}^{z} \frac{8 \mu}{\pi R^{4}}\left\{\int_{0}^{z} 2 \pi R \frac{\partial R}{\partial t} d z\right\}+c_{2}
\end{aligned}
$$

It is to be noted that $P=P_{0}$ at $z=0$. Therefore, $-P_{0}=0+c_{2}$

From (i) and (ii) we get that

$P_{0}-P=-\int_{0}^{z} \frac{8 \mu}{\pi R^{4}}\left\{\int_{0}^{z} 2 \pi R \frac{\partial R}{\partial t} d z\right\} d z$

Now at $z=L, P=P_{L}$. Hence,

$P_{0}-P_{L}=-\int_{0}^{L} \frac{8 \mu}{\pi R^{4}}\left\{\int_{0}^{L} 2 \pi R \frac{\partial R}{\partial t} d z\right\} d z$

Now the flux $Q(z, t)=Q_{L}$ at $z=L$. Hence,

$Q_{L}=-\int_{0}^{L} 2 \pi R \frac{\partial R}{\partial t} d z$

Integrating,

$Q_{L}=-2 \pi R \frac{\partial R}{\partial t} L$
This calculation was all about the gallbladder i.e. a cylinder starting from $z=0$ to $z=L$. Now there is another cylinder i.e. cystic duct. It starts from $z=L_{0}$ to $z=L_{1}$.

From equation (12) we have pressure at $z=L$ is

$-P_{L}=-\frac{8 \mu}{\pi r^{4}}\left\{\int_{0}^{L} 2 \pi R \frac{\partial R}{\partial t} d z\right\} L+c_{3}$

$=-\frac{8 \mu}{\pi r^{4}} 2 \pi r \frac{\partial r}{\partial t} \cdot \frac{L^{2}}{2}+c$

where $r=f(R)$

$P_{L}=\frac{8 \mu}{\pi r^{4}} 2 \pi r y \frac{L^{2}}{2}+c$

where $y=\int_{0}^{L} \frac{\partial R}{\partial t} d z$

Let us take that $P_{L}=P_{L_{0}}$ at $L=0$. Therefore,

$-P_{L_{0}}=0+c$

Hence,

$-P_{L}=-\frac{8 \mu}{\pi r^{4}} 2 \pi r y \frac{L^{2}}{2}-P_{L_{0}}$

i.e. $P_{L}-P_{L_{0}}=\frac{8 \mu}{\pi r^{4}} 2 \pi r y \frac{L^{2}}{2}$

Similarly for $z=L_{1}$. Assuming that $\frac{\partial R}{\partial t}=\frac{\partial R_{1}}{\partial t}$.

Therefore,

$P_{L_{1}}-P_{L 1_{0}}=\frac{8 \mu}{\pi r_{1}^{4}} 2 \pi r_{1} y \frac{L_{1}^{2}}{2}$

Therefore,

$\frac{P_{L}-P_{L_{0}}}{P_{L_{1}}-P_{L_{1}}}=\frac{\frac{8 \mu}{\pi r^{4}} 2 \pi r y \frac{L^{2}}{2}}{\frac{8 \mu}{\pi r_{1}^{4}} 2 \pi r_{1} y \frac{L_{1}^{2}}{2}}=\frac{L^{2} r_{1}^{3}}{L_{1}^{2} r^{3}}$

Hence,

$r_{1}^{3}=\frac{L_{1}^{2} r^{4}\left(P_{L}-P_{L_{0}}\right)}{L^{2}\left(P_{L_{1}}-P_{L_{0}}\right)}$

As we have $Q=-2 \pi R \frac{\partial R}{\partial t} z$

And at $z=L$, 
$Q_{L}=-2 \pi R \frac{\partial R}{\partial t} L$

Again at $z=L_{1}$,

$Q_{L_{1}}=-2 \pi R \frac{\partial R}{\partial t} L_{1}$ it is to be noted that $\frac{\partial R}{\partial t}=\frac{\partial R_{1}}{\partial t}$

Therefore,

$\frac{Q_{L}}{Q_{L_{1}}}=\frac{-2 \pi R \frac{\partial R}{\partial t} L}{-2 \pi R_{1} \frac{\partial R}{\partial t} L_{1}}=\frac{-R L}{R_{1} L_{1}}$

This gives

$R_{1}=\frac{-R L Q_{L_{1}}}{-L_{1} Q_{L}}$

Hence $\quad r_{1}=\frac{-r L Q_{L_{1}}}{-L_{1} Q_{L_{1}}}$

Here minus sign shows inverse relation in the quantity.

Putting this value of $r_{1}$ in (13) we get

$\frac{r^{3} L^{3} Q_{L_{1}}^{3}}{L_{1}^{3} Q_{L}^{3}}=\frac{L_{1}^{2} r^{4}\left(P_{L}-P_{L_{0}}\right)}{L^{2}\left(P_{L_{1}}-P_{L_{0}}\right)}$

Hence,

$\frac{Q_{L_{1}}^{3}}{Q_{L}^{3}}=\frac{L_{1}^{5} r\left(P_{L}-P_{L_{0}}\right)}{L^{5}\left(P_{L_{1}}-P_{L_{0}}\right)}$

It tells the behavior of flux with pressure difference.

This study was in the case of simple cylinder, a gallbladder. Although gallbladder is not proper cylinder, but flux is calculated in case of idealized cylinder.

In reference to the paper "One-Dimensional Models of the Human biliary System" by W.G. Li et.al. the comparison to study of gallbladder is done. In this model two cases are considered rigid wall model, elastic wall model. In both of the two cases two sub-cases are taken emptying phase, refilling phase.

In this model $\mathrm{T}$ junction between cystic duct and common bile duct and common bile duct is in consideration. In rigid wall model in emptying case the results are:

The pressure drop in the cystic duct is

$\Delta P_{C D}=\frac{128 \mu Q}{\pi d_{e q}^{4}} L_{e q}$

Where $L_{e q}$ is the equivalent length, and $d_{e q}$ is the equivalent diameter.
For common bile duct

$\Delta P_{C B D}=\frac{128 \mu Q}{\pi d_{C B D}^{4}} L_{C B D}+\Delta p_{t e}$

Where $\Delta p_{t e}$ accounts for $\mathrm{T}$ junction.

In refilling case pressure drop in $\mathrm{CBD}$ is same as (A), and for common hepatic duct is

$\Delta P_{C H D}=\frac{128 \mu Q}{\pi d_{C H D}^{4}} L_{C H D}+\Delta p_{t h}$

Where $\Delta p_{t h}=16 c_{4} \frac{\rho Q^{2}}{\pi^{2}}+16 c_{1} \frac{\rho Q^{2}}{\pi^{2} d_{C H D}^{4}}$

And the total pressure drop is

$\Delta P_{R F}=\frac{128 \mu Q}{\pi d_{e q}^{4}} L_{e q}+\frac{128 \mu Q}{\pi d_{C H D}^{4}} L_{C H D}+\Delta p_{t h}$

Combining results for cystic duct and common bile duct we get

$\frac{\Delta P_{C D}}{\Delta P_{C B D}}=\frac{\frac{128 \mu Q}{\pi d_{e q}^{4}} L_{e q}}{\frac{128 \mu Q}{\pi d_{C B D}^{4}} L_{C B D}}$

Here leaving T-junction term $\Delta p_{t e}$.

$\frac{\Delta P_{C D}}{\Delta P_{C B D}}=\frac{d_{C B D}^{4} L_{e q}}{d_{e q}^{4} L_{C B D}}$

Considering diameter $\mathrm{d}$ as a function of radius we get

$\frac{\Delta P_{C D}}{\Delta P_{C B D}} \propto \frac{L_{e q} f\left(R_{C B D}\right)}{L_{C B D} f\left(R_{e q}\right)}$

Now for two cylinders done earlier we have proved that

$f\left(R_{1}\right)=\frac{L_{1}^{2} f(R)\left(P_{L}-P_{L_{0}}\right)}{L^{2}\left(P_{L_{1}}-P_{L_{0}}\right)}$

As $P_{L}-P_{L_{0}}$ is the pressure difference term taking it as $\Delta p_{L}$ we get

$\frac{\Delta p_{L}}{\Delta p_{L_{1}}} \propto \frac{L^{2} f\left(R_{1}\right)}{L_{1}^{2} f(R)}$

Equivalently,

$\frac{P_{L}-P_{L_{0}}}{P_{L_{1}}-P_{L_{1}}}=\frac{L^{2} R_{1}^{3}}{L_{1}^{2} R^{3}}$ 
i.e

$\frac{\Delta P_{L}}{\Delta P_{L_{1}}} \propto \frac{L_{\text {eff }} R_{\text {leff }}}{L_{\text {leff }} R_{\text {eff }}}$

where, $L_{\text {eff }}$ and $R_{\text {eff }}$ are some functions of length and radius for one cylinder (gallbladder), similarly for cystic duct.

Now using value of flux from (12) into (13) we get

$\frac{Q_{L_{1}}^{3}}{Q_{L}^{3}}=\frac{L_{1}^{5} r\left(P_{L}-P_{L_{0}}\right)}{L^{5}\left(P_{L_{1}}-P_{L_{10}}\right)}$

It tells the behavior of flux with pressure difference.

It is clear that $(\mathrm{B})$ and $(\mathrm{C})$ are more or less similar. The terms and the interpretation for the case of gallbladder and combined effect of cystic duct and common bile duct is similar, i.e. the ratio of pressure difference in one cylinder/cystic duct to the other cylinder/common bile duct is inversely proportional to the ratio of radius and directly proportional to the ratio of lengths.

In the rigid wall model the walls of cystic duct are considered to be rigid. It is assumed that the common bile duct and the common hepatic duct are straight tubes and join at a T-junction. To model the effects of the cystic duct baffles on the flow, the baffles are arranged in the simplified manner.

The pressure drop during emptying is believed to have a link with the stone formation in gallbladder [10]. This is the reason why we tried to predict the pressure drop in a mathematical model of the human biliary system. It is noted that the key structure contributing to the pressure drop is the cystic duct, while the hepatic and common bile ducts offer little resistance or geometric changes during emptying and refilling. Therefore, to simplify the pressure drop prediction, the modeling focuses on the non-linear flow features in the cystic duct, while poiseuille flow is assumed in the other two biliary ducts.

It is clear that our results are in agreement with the similar model of W.G. Li et al. [10] This shows that terms and the interpretation for the case of gallbladder and combined effect of cystic duct and common bile duct is similar and the ratio of pressure difference in one cylinder or cystic duct to the other cylinder or common bile duct is inversely proportional to the ratio of radius and directly proportional to the ratio of lengths. These results are shown in graphs.

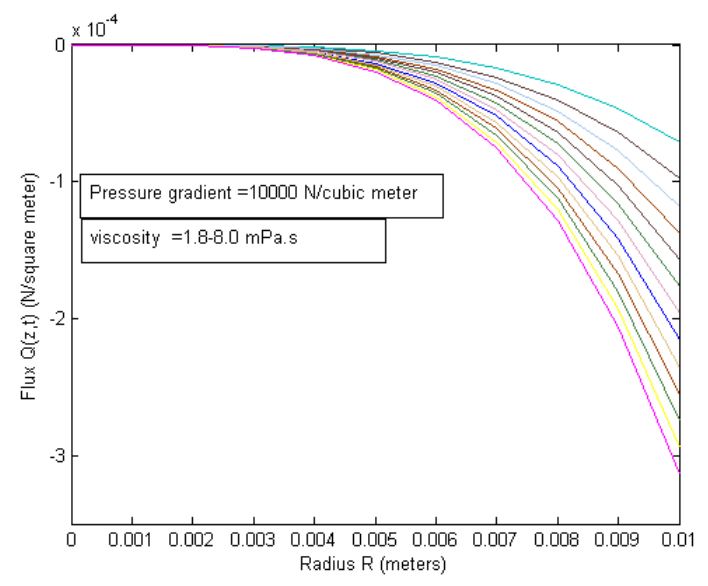

Fig. 1. Relationship between flux and radius of gallbladder and ducts

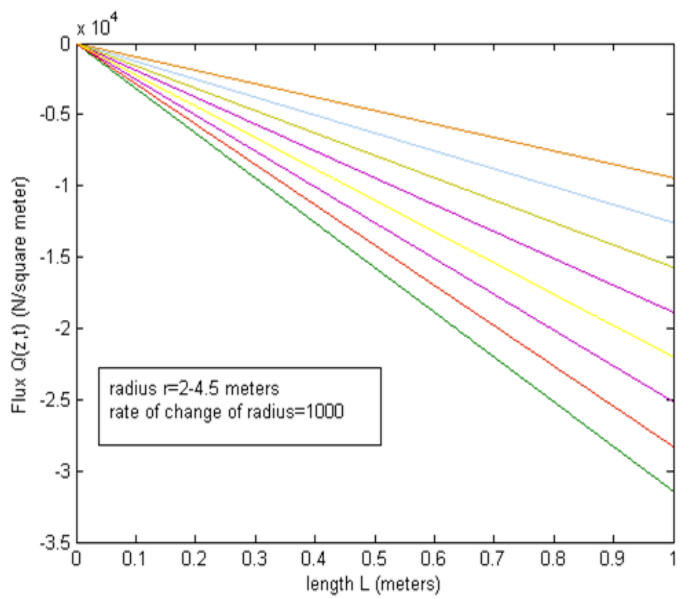

Fig. 2. Relationship between flux and length of the gallbladder and ducts

This study shows that the behavior of flow of bile in gallbladder is similar to the flow of bile in biliary tract, except for the $\mathrm{T}$-junction of biliary tract.

\section{CONCLUSION}

The present study tells the anatomy, functions, and diseases of the human biliary system. Along with that the idealized model, assuming cylindrical shape of the gallbladder is solved. It is clear that gallstones are very closely related to the model studied. Here all three ducts are assumed to be cylindrical in shape. For the first case of gallbladder, the amount of flux increases as radius decreases and vice versa. Hence for different human gallbladders the length varies and hence the flux. For a gallbladder of longer in shape the flow of bile is lesser. There are two phases of gallbladder due to contraction and extension. The flux or flow of bile in one case is inversely proportional to the pressure drop and pressure drop in a particular case is equivalent to the pressure difference. The results are fairly in agreement with the study of W.G.Li et. al [10]. From Fig. 1 and Fig. 2 it is observed that the flow of bile decreases on increasing the radius and length of gallbladder. Along with this (17) depicts that the pressure difference decreases on 
decreasing the effective length and increasing effective radius of cylinders. The third result i.e. a relationship between flux and pressure difference shows that for a fixed length on increasing pressure difference flux increases as natural phenomenon of bile. With the help of these symmetric results it can be easily observed that flow of bile in gallbladder is symmetric to the flow of bile in biliary tract and results are in agreement with the literature found.

\section{REFERENCES}

[1] G.E. Burget (1925), "The regulation of the flow of bile".

[2] Cz. M. Rodkiewicz (1978), "Empirical relationships for the flow of bile".

[3] Cz. M. Rodkiewicz,W.J.Otto (1979), "On the Newtonian behavior of bile".

[4] Norman A. Mazer and Martin C. Carey (1984), "Mathematical model of biliary lipid secretion: a quantitative analysis of physiological and biochemical data from man and other species".

[5] T V Taylor and C P Armstrong (1987), "Migration of gallstones".

[6] Dieter Jungst,Thomas Lang,Peter Huber, Volker Lange and Gustav Paumgartner (1993), “ Effect of phospholipids and bile acids on cholesterol nucleation time and vesicular/micellar cholesterol in gallbladder bile of patients with cholesterol stones".

[7] Dieter Jungst, Anna Niemeyer, Iris Muller,Benedikta Zundt, Gunther Meyer, Martin Wilhelmi, Reginald del Pozo (2001), "Mucin and phospholipids determine viscosity of gallbladder bile in patients with gallstones".

[8] Quang Dang, Hans Gregersen, birgitte duch and Ghassan s. Kassab (2004), "Indicial response functions of growth and remodeling of common bile duct postobstruction".

[9] N.C.Bird, R.C.Ooi, X.Y.Luo,S.B.Chin and A.G.Johnson (2006), "Investigation of the functional three-dimensional anatomy of the human duct: A single Helix?".

[10] W.G.Li,X.Y.Luo,A.G.Johnson,N.A.Hill,N.Bird,S.B. Chin (2007)," One dimensional Models of the Human Biliary System".

[11]Li, W.G., Luo, X.Y., Chin, S.B., Hill, N.A., Johnson, A.G. and Bird, N.C. (2008), "Non-Newtonian bile flow in elastic cystic duct:one- and threedimensional modeling".

[12] http://www.netdoctor.co.uk/diseases/facts/gallbladde rdisease.htm
Dharna Satsangi has earned integrated M.Sc.-M. Phil. in Mathematics with specialization in Computer Science from the Dayalbagh Educational Institute, India (2009) and is currently pursuing Ph.D. at the same department. Her research includes mathematical models of biological systems, psychological behavior of humans and graph layout problems.

Prof. Arun K. Sinha has earned M. Sc. in Mathematics Specialization in Statistics and Ph.D. in Mathematics from Agra College, India. His research areas are statistics and bio-mathematics. He has 35 years of teaching experience. Currently he is Head, Department of Mathematics, Dayalbagh Educational Institute, Agra. 